\title{
Radio Search for Mafife 1 Draws a Blank
}

IT is always exciting when something new is discovered on one's own doorstep, as it "were. Last January a group in the United States reported that a recently discovered infrared object is almost certainly an elliptical galaxy at a distance of three million light years or so, which places it at the edge of the Local Group of galaxies containing the Milky Way (see Nature, 229, 84 ; 1971). Known as Maffei 1 after its discoverer Paulo Maffei who reported it in 1968, the new object seems to be comparable in mass to the Milky Way. This makes Maffei 1 a significant new member of the Local Group that has hitherto gone unnoticed.

That the existence and nature of Maffei 1 have gone unrecognized for so long is not surprising, however. At a galactic latitude of $-0.5^{\circ}$, light from the object has to pass through the thickness of the Milky Way system before reaching the Earth, and the same goes for a second infrared object reported by Maffei in a similar direction. Obscuration by interstellar material trapped in the plane of the Milky Way is then a serious problem. Maffei 1 is therefore highly reddened, for the same reason that the setting Sun appears red through the thickness of the atmosphere. Hence Maffei 1 shows up well in the infrared, but is difficult to discern at visible wavelengths.

The story of Maffei 1 is taken a step further in this issue of Nature (page 103), from a radio astronomer's viewpoint. J. H. Oort, Leiden Observatory, gently points out that the absence of radio emission from Maffei 1 is going to be a problem. According to the original January article in Astrophysical Journal Letters, with an authorship reading like an extract from a Who's Who of astronomy, Maffei 1 is to be regarded, probably, as a giant elliptical galaxy at a distance between $0.3 \mathrm{Mpc}$ (megaparsec) and $4 \mathrm{Mpc}$ (1 parsec $=3.26$ light years). A distance of $1 \mathrm{Mpc}$ is accepted as a reasonable estimate (Spinrad et al., 163, L25 ; 1971). This places it at the edge of the Local Group. The mass of Maffei 1 is determined to be comparable to M 31 (the Andromeda nebula, at a distance of $700 \mathrm{kpc}$ ), and the Milky Way, the two normal spiral members of the Local Group. Elliptical galaxies normally do not contain any recognizable structure-their brightness decreases from the centre towards the edges, and the isophotes are circles or ellipses. Oort's most telling point then is that Maffei 1 is at least four magnitudes fainter at radio wavelengths than the faintest giant elliptical from which radio emission has so far been detected.

Oort's article has the distinction of being the first scientific article to come out of the new radio telescope array at Westerbork, Holland, officially opened on June 24 last year (see Nature, 226, $1189 ; 1970$ ). Chiefly the array of ten dishes is for detailed mapping of the radio sky, working on the aperture synthesis principle. What Oort is now presenting is a map of the $21 \mathrm{~cm}$ radio emission from an area of sky including Maffei 1. At the position of Maffei 1 there is no detectable radio emission greater than the order of 0.001 flux units. Taking the distance of $1 \mathrm{Mpc}$ favoured by Spinrad $e t$ al., this leads to an upper limit for the total radio energy of the order of $10^{34} \mathrm{erg} \mathrm{s}^{-1}$.

This result may be an embarrassment because the giant ellipticals that have been detected at radio wavelengths have significantly higher total radio energies. Oort has taken a look at two radio surveys which include an examination of elliptical galaxies, and points out that giant ellipticals from which radio emission has been detected have radio energies greater than $5 \times 10^{38} \mathrm{erg} \mathrm{s}^{-1}$, four orders of magnitude greater than the upper limit for Maffei 1. But the surveys from which Oort has determined this figure were not sensitive to fluxes corresponding to total radio energies of less than of the order of $10^{39} \mathrm{erg} \mathrm{s}^{-1}$. Clearly then, as Oort points out, the contrast between Maffei 1 and other giant elliptical galaxies has to be taken with a grain of salt ; the population of giant ellipticals could well extend to members having radio energies of the order of $10^{34} \mathrm{erg} \mathrm{s}^{-1}$, and this component would have remained undetected by the surveys. But Oort and his group at Westerbork have become sufficiently interested in the problem to continue their survey so as to achieve a sensitivity limit lower than the previous surveys.

Otherwise the evidence for Maffei 1 being a giant elliptical galaxy is compelling. Spinrad et al. base their conclusion on a battery of tests carried out at the Leuschner, Lick and Hale observatories. Briefly, the arguments are that the ellipticity of the system can be traced out in the isophotes to a radius of 3' or more; after allowing for reddening the spectrum is what one would expect from giant ellipticals; the infrared flux from Maffei 1 is comparable with that detected from the centre of M 31; and the spectrum contains absorption lines that are commonly found in the nuclei of certain types of galaxies including ellipticals. The distance estimate is thought to be too crude to be accepted as part of the evidence for the extragalactic nature of the object, but the rest of the evidence builds up a strong case. Infrared astronomy could well turn up further missing members of the Local Group.

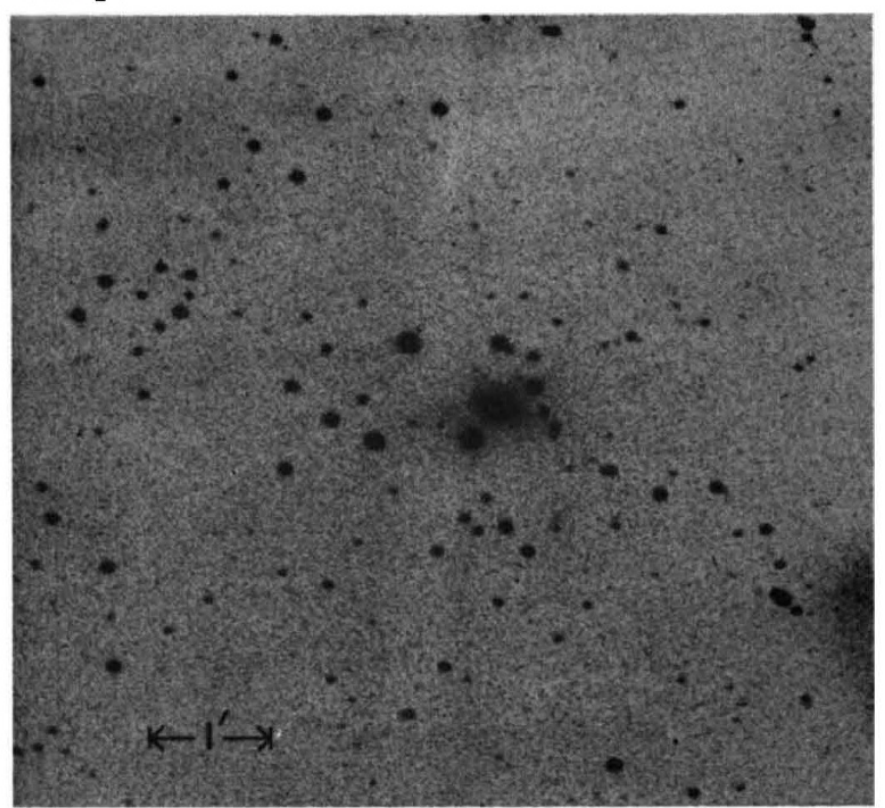

The diffuse object is Maffei 1, observed with the Leuschner 30-inch telescope (plate L2, Astrophys. J. Lett., 163 ; 1971). 